\title{
SOME REMARKS ON THE VALIDITY OF THE PRINCIPLE OF EXPLOSION IN INTUITIONISTIC LOGIC ${ }^{1}$
}

\author{
Edgar Campos ${ }^{2} \&$ Abilio Rodrigues ${ }^{3}$
}

\begin{abstract}
The formal system proposed by Heyting $(1930,1936)$ became the standard formulation of intuitionistic logic. The inference called ex falso quodlibet, or principle of explosion, according to which anything follows from a contradiction, holds in intuitionistic logic. However, it is not clear that explosion is in accordance with Brouwer's views on the nature of mathematics and its relationship with logic. Indeed, van Atten (2009) argues that a formal system in line with Brouwer's ideas should be a relevance logic. We agree that explosion should not hold in intuitionistic logic, but a relevance logic requires more than the invalidity of explosion. The principle known as ex quodlibet verum, according to which a valid formula follows from anything, should also be rejected by a relevantist. Given ex quodlibet verum, the inference we call weak explosion, according to which any negated proposition follows from a contradiction, is proved in a few steps. Although the same argument against explosion can be also applied against weak explosion, rejecting the latter requires the rejection of ex quodlibet verum. The result is the loss of at least one among reflexivity, monotonicity, and the deduction theorem in a Brouwerian intuitionistic logic, which seems to be an undesirable result.
\end{abstract}

\section{Introduction: Brouwer on mathematics and logic}

The main divergence between Brouwer and the standard approach to mathematics, based on classical logic, is his understanding of the notions of existence and truth as applied to mathematics. According to Brouwer, "to exist in mathematics means: to be constructed by intuition" (Brouwer, 1907, p. 96). So, the existence of mathematical objects such as numbers, functions, and sets depends on some construction introspectively carried out by some conscious subject.

The basic intuition of mathematics is the passage of time, which provides the raw material for the mathematical constructions and poses restrictions on which constructions may or may not be carried out (Brouwer, 1907, p. 52). The basic idea is that the human mind perceives the flow of time, the sequence of moments, one after another. A first moment is followed by a second, and this is something like a 'pure form of time' - indeed, an idea akin to Kantian pure intuition of time. The concept of a sequence of numbers is based on this basic intuition (Brouwer, 1912, pp. 127-128). The truth of any assertion about these mathematical objects depends on these constructions: "truth is only in reality i.e. in the present and past experiences of consciousness" (Brouwer, 1948, p. 1243).

1 The first author acknowledges support from CAPES (Coordenação de Aperfeiçoamento de Pessoal de Nível Superior), a doctorate scholarship. The second author acknowledges support from $C N P q$ (Conselho Nacional de Desenvolvimento Científico e Tecnológico), research grant $311911 / 2018-8$. The authors would like to thank Rodolfo Ertola-Biraben, André Porto and Wagner Sanz for some helpful comments and suggestions. They are not responsible, however, for any infelicities that may remain.

2 Department of Philosophy, Federal University of Minas Gerais. Email: ehncampos@gmail.com.

${ }^{3}$ Department of Philosophy, Federal University of Minas Gerais. Email: abilio.rodrigues@gmail.com.

DOI : 10.21452/LnA_serie_n_v01_book_seminario-logica-no-aviao-2013-2018_campos-rodrigues_p.29-43 
A consequence of such a view is his conception of logic being dependent upon mathematics. So conceived, logic just describes patterns of mental constructions in the language of mathematics, and the latter is a linguistic representation of these mental mathematical constructions. A valid formula in a Brouwerian intuitionistic logic represents something that can be constructed from the fundamental intuition of mathematics, and when a mathematician writes down a proof, such a proof is a linguistic description of those mental constructions.

Brouwer developed his well-known criticism of the principle of excluded middle, double negation elimination, and other classically valid principles, based on his views on the nature of mathematics and its relationship with logic. Excluded middle is not reliable because "it claims that every supposition is either true or false" (Brouwer, 1908, p. 109), which means, given that mathematical truth amounts to being mentally constructed, that every mathematical problem can be solved. The fact that there are open problems in mathematics implies that there is a proposition $A$ such that no construction of $A$, nor of $\neg A$ has been carried out; therefore, there is no guarantee that $A \vee \neg A$ represents a mathematical construction.

In the standard formalization of intuitionistic logic (Heyting, 1930, 1956), the inference known as ex falso quodlibet, or the principle of explosion,

$$
\text { (EXP) } A, \neg A \vdash B \text {, }
$$

holds. The explanation of the meanings of the intuitionistic connectives, given by the $B H K$-interpretation, also takes EXP as valid (Troelstra and van Dalen, 1988). In the minimal logic proposed by Johansson (1937), although EXP does not hold, a weaker principle, that we call here weak explosion, holds:

$$
(W-E X P) A, \neg A \vdash \neg B .
$$

In both intuitionistic and minimal logic, and in classical logic as well, the principle known as ex quodlibet verum,

$$
(E Q V) \vdash A \rightarrow(B \rightarrow A),
$$

holds. $E Q V$ is tantamount, in natural deduction systems, to the vacuous discharge of an assumption, and in sequent calculus, to the structural rule of weakening. The validity of $W$-EXP depends essentially on EQV.

The validity of EXP within the framework of a Brouwerian logic was criticized and rejected by Kolmogorov (1925) in his first work on intuitionistic logic, and also by Johansson (1937), where we find the formal system that became known as minimal logic. According to Haack (1974), Heyting's justification of EXP in intuitionistic logic extends the intuitionistic sense of 'construction' in such a way that it could no longer be regarded as intuitionistic. As a result, she thinks that minimal logic is better to represent a Brouwerian intuitionistic logic than Heyting's proposal (Haack, 1974, p. 102).

van Atten (2009) also defends the view that EXP should not be valid within a constructivist framework. However, van Atten goes further and argues that a formal system in line with Brouwer's ideas should be a relevance logic. But if this were the case, not only $E X P$ but also EQV and $W$-EXP should be considered invalid. Explosion, the deduction theorem and $E Q V$ yield the so-called paradoxes of material implication, and avoiding the latter is a motivation for relevance logics (see, for example, Mares, 2018). A relevantist, of course, also rejects the validity of $W$-EXP.

In this paper, we discuss the validity of $E X P, W$-EXP, and $E Q V$ in the framework of a Brouwerian intuitionistic logic. We agree with van Atten (2009) and Haack (1974) that explosion should not hold. On the other hand, although there may be some debate regarding the validity of $W-E X P$ and $E Q V$, both are much more plausible than explosion 
in a constructive framework. We also suggest a normative justification for $W$-EXP in constructive mathematics as a way to prohibit contradictions. Although this idea does not fit with Brouwer's descriptive conception of logic, and cannot be found in his works, given the assumption that mathematics is consistent, $W$-EXP is more effective than the principle of non-contradiction in avoiding inconsistency.

The remainder of this text is structured as follows. In Section 2, we see Brouwer's remarks on the hypothetical judgment (implication) and the ocurrence of contradictions. In Section 3, the passages in Kolmogorov (1925, 1932), Heyting (1930, 1934, 1956), Glivenko $(1928,1929)$ and Johansson (1937) where EXP is mentioned are presented, and we compare their views with Brouwer's remarks on implication and contradiction. In Section 4, we discuss how the $B H K$-interpretation justifies EXP. Section 4 also discusses the validity of disjunctive syllogism, $W$-EXP, and $E Q V$ in the framework of a Brouwerian intuitionistic logic.

\section{The hypothetical judgment}

The discussion about the validity of EXP depends on Brouwer's views on the implication. An implication $A \rightarrow B$ is to be understood as a kind of 'path' that leads from $A$ to $B$. So, whether or not EXP should be considered as valid, according to Brouwer's views depends on how any proposition $B$ can be obtained from a pair of contradictory propositions $A$ and $\neg A$. We divide the passage where Brouwer presents his views on implication and contradictions into two parts, that we call $I$ and $I I$.

\section{$I$}

In one particular case the chain of syllogisms is of a somewhat different kind, which seems to come nearer to the usual logical figures and which actually seems to presuppose the hypothetical judgment from logic. This occurs when a structure is defined by some relation in another structure, while it is not immediately clear how to effect its construction. Here it seems that the construction is supposed to be effected, and that starting from this hypothesis a chain of hypothetical judgments is deduced. But this is no more than apparent; what actually happens is the following: one starts by setting up a structure which fulfills part of the required relations, thereupon one tries to deduce from these relations, by means of tautologies, other relations, in such a way that these new relations, combined with those that have not yet been used, yield a system of conditions, suitable as a starting-point for the construction of the required structure. Only by this construction will it be proved that the original conditions can be fulfilled. (Brouwer, 1907, p. 72-73)

By a 'hypothetical judgment from logic' Brouwer means a sequence of one or more inferences that start from an assumption $A$ and reach a conclusion $B$. The result of such a reasoning is an implication $A \rightarrow B$. Brouwer adds:

\section{II}

'But', the logician will retort, 'it might have happened that in the course of these reasonings a contradiction turned up between the newly deduced relations and those that had been kept in store. This contradiction, to be sure, will be observed as a logical figure, and this observation will be based upon the principium contradictionis.' To this we can reply: 'The words of your mathematical demonstration merely accompany a mathematical construction that is effected without words. At the point where 
you enounce the contradiction, I simply perceive that the construction no longer goes, that the required structure cannot be imbedded in the given basic structure. And when I make this observation, I do not think of a principium contradictionis. (Ibidem)

In $I$, Brouwer talks about implication, and in $I I$, about the principle of noncontradiction. These passages, especially $I$, yield some interpretation issues. We start with the easiest one, quotation $I I$.

2.1. The principle of non-contradiction. We want to draw your attention to two points in quotation II. The first is that a contradiction, as expected, given the assumption that mathematics is consistent, cannot be constructed. So,

the principle of contradiction is indisputable: The results that we perform the imbedding of a system $a$ into a system $b$ in a prescribed manner, and that we are arrested by the impossibility of such an imbedding, exclude each other (Brouwer, 1908, p. 109).

A contradiction is the sign that the intended construction cannot go further. Hence, the main role for a contradiction is to show that a hypothetical reasoning has reached a dead end and has to stop. Note that a contradiction can only appear from a reasoning that starts from premises, that is, a hypothetical reasoning. This is the basis of the intuitionistic interpretation of negation defined in terms of the primitive notion of absurd, $A \rightarrow \perp$.

The second is the claim that logic is secondary to the mental constructions carried out by the mathematician. It is not that a construction cannot be done because it disobeys non-contradiction. Quite the contrary, non-contradiction holds because no construction of a contradiction can be done. The principle of non-contradiction $\neg(A \wedge \neg A)$, and the notion of absurd, represented by $\perp$, are just linguistic representations of mental mathematical constructions that cannot go further.

2.2. Implication. Now, we turn to quotation $I$, where Brouwer presents his views on implication. van Dalen (2004) proposed the following reading:

Brouwer's comments on hypothetical judgments are unfortunately rather cryptic. They can be read in various ways. The most plausible reading seems to be the following.

$(\alpha)$ In order to establish $A \rightarrow B$, one has to carry out two tasks, namely, (i) find a construction for (the structure specified by) $A$, (ii) find a construction for (the structure specified by) $B$ that departs from the first construction. We have left the reference to the "embedding" implicit. In fact, this embedding is mostly tacitly incorporated into the construction for B (van Dalen, 2004, p. 251).

Although van Dalen says in the quotation above that this reading is the 'most plausible', he will later reject this (van Dalen, 2008, p. 20). His interpretation was indeed criticized by van Atten (2009), who proposed the following interpretation:

$(\beta)$ In order to establish $A \rightarrow B$, one has to conceive of $A$ and $B$ as conditions on constructions, and to show that from the conditions specified by $A$ one obtains the conditions specified by $B$, according to transformations whose composition preserves mathematical constructibility (van Atten, 2009, p. 128). 
As van Atten points out, the central aspect of interpretation $\beta$ is the notion of condition: "Brouwer avoids commitment to hypothetical constructions by considering, not constructions, but conditions on constructions", and such conditions are "actual and therefore ontologically unproblematic" (van Atten, 2009, p. 127).

According to $\alpha$, an implication $A \rightarrow B$ implies that both $A$ and $B$ have been effectively constructed: first, a construction of $A$ has to be already done, and so $B$ is constructed departing from $A$. Thus, $\alpha$ excludes the possibility of putting forward $A$ as a mere hypothesis. Notice that this reading makes it impossible even to prove $\neg A$, since such a proof would need an effective construction of $A$, which is precisely what cannot be done when $\neg A$ holds.

The interpretation $\beta$, on the other hand, understands $A$ as a condition on a construction, and so, unlike $\alpha$, does not require that $A$ has been effectively constructed. So, a condition for a construction can be, so to speak, just 'imagined', and what can be done from these conditions is obtained by means of steps that go from one condition to another condition. Thus, an implication $A \rightarrow B$ can be read as 'if the condition $A$ is satisfied, then the condition $B$ is satisfied', and if eventually it is proved that condition $B$ cannot be satisfied, i.e., $\neg B$ is constructed, we can draw the conclusion that condition $A$ cannot be satisfied either, so $\neg A$. These reasonings are clearly constructive. This interpretation also acquires support from a claim made by Brouwer in one of his last publications, where he talks about a 'constructional condition' on a mathematical system:

the wording of a mathematical theorem has no sense unless it indicates the construction either of an actual mathematical entity or of an incompatibility (e.g., the identity of the empty two-ity with an empty unity) out of some constructional condition imposed on a hypothetical mathematical system (Brouwer, 1954, p. 3).

The rejection of $E X P$ by interpretation $\alpha$ is straightforward. If an implication $A \rightarrow B$, in order to be a legitimate mathematical construction, requires that a construction of the antecedent $A$ has been successfully carried out, and since a construction of a contradiction is never carried out, clearly nothing can be constructed from a contradiction. The interpretation $\beta$ also rejects the validity of $E X P$. Indeed, it is not clear how to understand a contradiction as a condition of a construction that, in the end, would obtain an arbitrary proposition $B$, or better, an arbitrary condition expressed by a proposition $B$. Therefore, both interpretations of Brouwer's remarks on the hypothetical judgment provide reasons for rejecting the validity of EXP in a Brouwerian intuitionistic logic. ${ }^{4}$

\section{Kolmogorov, Heyting, Glivenko, Johansson}

As we have seen, Brouwer has not provided any reasons for accepting EXP in his works, actually, quite the contrary, we can find strong evidence for rejecting it. However, EXP holds in the formalization of intuitionistic logic that became standard. Different formal systems, conceived to express Brouwer's ideas, were proposed by Kolmogorov (1925), Glivenko (1928, 1929), and Heyting (1930, 1956). In Johansson (1937) a formalization of intuitionistic logic was proposed, although he did not explicitly mention Brouwer's work.

3.1. Kolmogorov 1925. The first formal system designed to express Brouwer's ideas was proposed by Kolmogorov (1925), in a language with only negation and implication.

${ }^{4}$ van Atten (2009, p. 130) reaches a similar conclusion: "we cannot expect to be able to come up with the required transformation for just any given pair of a false antecedent and a consequent, let alone that we have a uniform method to do this. As a consequence, there is on this account of the hypothetical judgement no ground to accept the Ex Falso principle $[E X P]$ in its full generality." 
He proposed a propositional logic by dropping the two axioms of negation presented in Hilbert (1923),

$$
A \rightarrow(\neg A \rightarrow B),
$$

and

$$
(A \rightarrow B) \rightarrow((\neg A \rightarrow B) \rightarrow B)
$$

and adding only one axiom for negation, that we call here introduction of negation,

$$
(I N)(A \rightarrow B) \rightarrow((A \rightarrow \neg B) \rightarrow \neg A),
$$

that correctly expresses the notion of intuitionistic negation (Section 2.1 above). In Kolmogorov's system, $E Q V$ holds and $W$-EXP can be proved. About the rejection of EXP, Kolmogorov remarks that:

Hilbert's first axiom of negation, 'Anything follows from the false' (...) does not have and cannot have any intuitive foundation since it asserts something about the consequences of something impossible: we have to accept $B$ if the true judgment $A$ is regarded as false (Kolmogorov, 1925, p. 421).

Thus, in 1925, Kolmogorov defended the view that it makes no sense to speak about what would be constructed having as a starting point something that cannot be constructed. Notice that this claim is in line with the argument for the rejection of EXP based on Brouwer's views, discussed in Section 2 above. Later, however, Kolmogorov changed his mind. In 1932, when he presents the interpretation of propositional connectives in terms of solutions of problems, he says that

the calculus of problems is formally identical with the Brouwerian intuitionistic logic, which has recently been formalized by Mr. Heyting (Kolmogorov, 1932, p. 328).

He refers to Heyting (1930), where a formal system for intuitionistic logic, in which EXP holds, has been presented (Section 3.2 below). Note that there is no evidence that Kolmogorov's article published in 1925 was known by Heyting before the latter conceived his formal system. Regarding the justification of the validity of $E X P$, now we read "As soon as $\neg a$ is solved, then the solution of $a$ is impossible and the problem $a \rightarrow b$ is without content" (Kolmogorov, 1932, p. 331). This interpretation intends to validate the principle of explosion because it presupposes that "the proof that a problem is without content will always be considered as its solution" (Kolmogorov, 1932, p. 329). So, once $\neg a$ has been solved, $a \rightarrow b$ is also considered solved. ${ }^{5}$ This interpretation is not implausible, but we do not think that it fits Brouwer's constructivist ideas.

3.2. Heyting 1930. Heyting presented his version of intuitionistic logic during a prize contest promoted by the Dutch Mathematical Society in 1927. Although the original manuscript has not survived, a revised version was published in 1930. This system, presented in a language with $\wedge, \vee, \rightarrow$, and $\neg$, contains the axiom

$$
4.1 \neg a \rightarrow(a \rightarrow b) \text { (Heyting, 1930, p. 313). }
$$

Heyting's system became the standard intuitionistic logic, either with $\neg$ as primitive, or with $\perp$ as primitive and negation $\neg A$ defined as $A \rightarrow \perp$.

This work was favorably received by Brouwer, as it is clear from his letter to Heyting, dated July 17th, 1928 (van Dalen, 2011, p. 333-334). We do not think, however, that such positive reception of Heyting's paper by Brouwer should be taken as evidence that the latter would accept the validity of EXP. Supporting a publication does not imply

\footnotetext{
${ }^{5}$ We use here capital letters as propositional variables. However, we keep the notation used by the authors in the quotations, and in our comments related to such quotations.
} 
the complete agreement with its contents - indeed, in the 1940s, Brouwer supported Griss' publications on negationless mathematics, even though he managed to publish a counterexample to Griss' ideas.

Heyting provides an informal explanation for the meaning of the implication that intends to justify both $E Q V$ and EXP:

The case is conceivable that after the statement $a \rightarrow b$ has been proved in the sense specified, it turns out that $b$ is always correct. Once accepted, the formula $a \rightarrow b$ then has to remain correct; that is, we must attribute a meaning to the sign $\rightarrow$ such that $a \rightarrow b$ still holds. The same can be remarked in the case where it later turns out that $a$ is always false (Heyting, 1930, p. 313).

Heyting's argument, however, as Johansson will point out in 1937 (Section 3.4 below), is not sound. He was thinking of a situation in which the proof of $A \rightarrow B$ was available, and subsequently a proof of $\neg A$ was obtained. But what EXP says is something rather different, and stronger, namely, that a proof of $\neg A$ automatically yields a proof of $A \rightarrow B$. Heyting clearly fails to justify this.

3.3. Glivenko 1928. In 1928, before the revised version of his 1927 paper was published by Heyting, Glivenko (1928), motivated by criticisms directed to Brouwer's intuitionism that he thought were mistaken, proposed a list of formulas that should be valid according to Brouwer's views. In this list, nothing equivalent to EXP appears. But in the following year, Glivenko added more axioms to the previous list of formulas of 1928 in order to "lay the complete foundations of a logical calculus" (Glivenko, 1929, p. 302). Among these axioms, we find $(\mathrm{C})$ and $(\mathrm{D})$ :

(C) $p \rightarrow(q \rightarrow p)$,

(D) $\neg q \rightarrow(q \rightarrow p)$.

He then asks about the validity of these axioms and provides the following explanation:

Are these axioms $\mathrm{C}$ and $\mathrm{D}$ admissible in the Brouwerian logic? I will confine myself here to a simple remark. By virtue of axioms VI and VII [resp. $p \rightarrow(p \vee q)$ and $q \rightarrow(p \vee q)$ ], expressions $\mathrm{C}$ and $\mathrm{D}$ are nothing more than the immediate consequences of the principle:

$$
(p \vee \neg q) \rightarrow(q \rightarrow p)
$$

whose admissibility is quite evident, because the formal implication $p \rightarrow q$ has no other sense than "when one accepts the truth of $p$, one must accept that of $q$ " (Glivenko, 1929, p. 302). ${ }^{6}$

The principle $(p \vee \neg q) \rightarrow(q \rightarrow p)$ is equivalent, given the deduction theorem, to the inference known as the disjunctive syllogism. Indeed, if disjunctive syllogism holds, EXP and $E Q V$ are easily proved. However, it is far from clear that disjunctive syllogism should be valid in intuitionistic logic - actually, we argue in Section 4.1 below that it should not be valid.

\footnotetext{
${ }^{6}$ In a footnote (Ibidem pp. 304-305), Glivenko remarks that it was Heyting who made him acknowledge the appropriateness of EXP in the Brouwerian logic and refers the reader to Heyting's revised version of his formalization that was going to be published in the Mathematische Annalen. However, because of Brouwer's dismissal of the Journal's editorial board by Hilbert in 1928, the episode that became known as the Grundlagenstreit, Heyting's article was published elsewhere.
} 
3.4. Johansson 1937. In Johansson (1937) a formal system that became known as minimal logic was presented, in a language with $\vee, \wedge, \rightarrow$, and $\neg$, whose only axiom for negation is the introduction of negation, expressed as

$$
4.11((a \rightarrow b) \wedge(a \rightarrow \neg b)) \rightarrow \neg a \text { (Johansson, 1937, p. 121). }
$$

Note that this axiom is equivalent, in a language with $\wedge$, to the axiom $I N$ proposed by Kolmogorov (1925) (see Section 3.1 above). Indeed, it is well-known that Kolmogorov's system is the fragment of minimal logic in a language with $\rightarrow$ and $\neg$. Johansson opens his article by mentioning two formulas accepted by Heyting (1930),

$$
2.14 b \rightarrow(a \rightarrow b),
$$

and

$$
4.1 \neg a \rightarrow(a \rightarrow b),
$$

and casts doubt on the validity of them. The first is $E Q V$, the second is tantamount to EXP. Regarding 4.1, he says that the case in which one writes $a \rightarrow b$ when $a$ is false or absurd,

means a difficult-to-overlook extension of the concept of [logical] consequence. It will be worth the effort to investigate if one cannot avoid it (Johansson, 1937, p. 119, our translation).

He ends up accepting $2.14(E Q V)$ but rejecting 4.1 (and so EXP) in his minimal logic. Johansson did not justify in this paper the rejection of $E X P$, but he expressed his concerns about EXP in a letter to Heyting dated September 23rd, 1935, prior to the 1937 article:

I have not been able to make my peace with Axiom 4.1. You say that when $a \rightarrow b$ has been proved, and later $\neg a$ is proved, then $a \rightarrow b$ should remain correct. Indeed; but $\neg a \rightarrow(a \rightarrow b)$ means that when $\neg a$ has been proved, $b$ at once becomes derivable from $a$, even when this had not been proved before. And that contradicts my intuition in the most violent way (Letter from Johansson to Heyting, apud van Atten, 2017).

We think that Johansson's argument above succeeds in refuting Heyting's justification of EXP (Section 3.2 above).

3.5. Heyting 1956. In 1955, a year before the publication of his Intuitionism: an Introduction, Heyting adds a short note to the French translation of Heyting (1934):

JOHANSSON [1] developed a 'minimal calculus' in which he adopts all the axioms from HEYTING except $\neg a \rightarrow(a \rightarrow b)$ : one may consider this calculus as the expression of the intuitionistic logic with another interpretation of the implication (Heyting, 1955, p. 19, our translation).

As van Atten (2017, Sec. 6.1) suggests, the conversation with Johansson may have had an effect on Heyting's position regarding EXP. In Heyting (1956), an effort is made to justify EXP along Brouwerian lines as much as possible:

Axiom X $[\neg p \rightarrow(p \rightarrow q)]$ may not seem intuitively clear. As a matter of fact, it adds to the precision of the definition of implication. You remember that $p \rightarrow q$ can be asserted if and only if we possess a construction which, joined to the construction $p$, would prove $q$. Now suppose that $\neg p$, that is, we have deduced a contradiction from the supposition that $p$ were carried out. Then, in a sense, this can be considered as a construction, which, joined to a proof of $p$ (which cannot exist) leads to a proof of $q$. I shall interpret the implication in this wider sense (Heyting, 1956, p. 106). 
It seems that Heyting wants to justify EXP along the same lines as he did for the other axioms of intuitionistic logic, namely, by presenting a construction that allows one to obtain a proof of an arbitrary proposition $q$ from any proof of $p$, once $\neg p$ has been constructed. But one can hardly take his attempt as successful. It is difficult to understand, in Brouwerian terms, how a construction of $\neg p$ together with a non-existent construction of $p$ could yield a construction of an arbitrary $q$.

\section{Explosion in the BHK-interpretation}

After Heyting's last effort, we find other attempts at the justification of EXP in intuitionistic logic. One comes from the standard proof interpretation for the logical constants, developed from the contributions of Brouwer, Heyting and Kolmogorov, known as the $B H K$-interpretation. The origins of the $B H K$-interpretation can be traced back to Kolmogorov's proposal of interpreting intuitionistic logic as being concerned with solutions of problems. Heyting (1956, p. 102) explains the intuitionistic connectives in terms of assertibility conditions. Later, Troelstra and van Dalen (1988) presented what became the standard $B H K$-interpretation in terms of proofs. They explain the validity of EXP as follows:

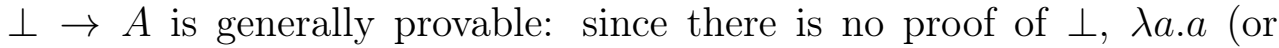
any other mapping) may count as a proof of $\perp \rightarrow A$, since it has to be applied to an empty domain. The principle $\perp \rightarrow A$ ("ex falso sequitur quodlibet") has sometimes been rejected as non-constructive (Johansson, 1937); (Heyting, 1956, 7.1.3) regards it as an extra stipulation fixing the meaning and use of $\perp, \rightarrow$ Troelstra and van Dalen (1988, p. 10).

Notice that van Dalen and Troelstra consider Heyting's explanation (quoted above in Section 3.5) as a stipulation that fixes the meaning of $\perp$ and $\rightarrow$, or $\neg$ and $\rightarrow$, depending on what one chooses as primitive. But if this inference were in accordance with Brouwer's ideas, it would not have to be the result of a stipulation. As far as we know, nowhere in Brouwer is there evidence in support of the claim that any operation (i.e., a construction), applied to an empty domain (i.e., to a non-existent proof of $\perp$ ) yields as a result a proof of an arbitrary proposition $A$.

Moreover, we think that this explanation has a circular ingredient. The argument that goes from 'there is no proof of $\perp$ ' to 'any given proof of $\perp$ can be transformed in a proof of $A$ ' seems to assume EXP in the meta-theory. There is a striking similarity between the claim that any operation applied to nothing yields anything and, for example, the proof that the empty set is a subset of any set, which uses explosion. Given that $\forall x \neg(x \in \emptyset)$, it follows that $\forall x \forall A(x \in \emptyset \rightarrow x \in A)$. It holds precisely because the antecedent $x \in \emptyset$ is false for any $x$. Classical logic, of course, explicitly relies on a classical meta-theory for the explanation of all the connectives. But it is doubtful that a Brouwerian intuitionistic logic should do the same regarding an inference that has no clear justification when we consider Brouwer's views.

4.1. Disjunctive syllogism. We saw, in Section 3.3 that Glivenko (1929) justifies the intuitionistic validity of EXP based on the fact that it follows from disjunctive syllogism:

(DS) $A \vee B, \neg A \vdash B$.

Indeed, there is a well-known proof of the principle of explosion that can be traced back at least to medieval times:

$$
\frac{\frac{A}{A \vee B} \vee I \neg A}{B} D S
$$


This proof makes essential use of disjunctive syllogism $(D S)$. van Atten (2009) argues that although EXP should be invalid within a constructive framework, there is no problem with disjunctive syllogism in a Brouwerian logic. He argues that the inference rules applied in the argument are indeed correct from a Brouwerian point of view, but the whole argument, from $A$ and $\neg A$ to $B$, is unreliable because there is no guarantee that the composition of the inference rules preserves constructibility (van Atten, 2009, p. 124). In other words, constructibility is not transitive, which means that a logic suitable for constructive mathematics cannot be transitive.

It is well-known that given a few assumptions $E X P$ and $D S$ are equivalent. Indeed, given $D S, E X P$ can be proved with introduction of disjunction (proof above), and given $E X P, D S$ can be proved with elimination of disjunction:

$$
\frac{A \vee B \frac{[A]^{1} \neg A}{B} \operatorname{EXP} \quad[B]^{1}}{B} \vee E, 1
$$

Now, since the validity of both $\vee I$ and $\vee E$ do not conflict with Brouwer's ideas, the rejection of EXP should imply the rejection of $D S$. The constructive validity of $D S$ has also been discussed by Tennant (1987). Both van Atten (2009) and Tennant rejected EXP and accepted $D S$ as valid, but the price paid is the loss of transitivity. We think, however, that there is no reason to reject transitivity in the framework of mathematics, including constructive mathematics. Brouwer agrees with this idea, as the following passage clearly shows:

[the inference] that concludes from the imbedding of a system $b$ into a system $c$, joined to the imbedding of a system $a$ into the system $b$, to a direct imbedding of the system $a$ into the system $c \ldots$ is nothing more than a tautology (Brouwer, 1908, p. 109).

By being 'nothing more than a tautology' Brouwer means that this inference, namely, transitivity, is constructively valid.

In intuitionistic logic, a disjunction $A \vee B$ holds if and only if $A$ has been constructed or $B$ has been constructed. But since $\neg A$ (the minor premise of $D S$ ) has been constructed, a construction of $A$ is not possible. Therefore, it has to be the case that the disjunction $A \vee B$ was the result of $\vee I$ from the premise $B$, and not from premise $A$. This is the same line of reasoning as found in van Atten (2009, p. 124). Nevertheless, rather than showing that $D S$ is valid, in our view, it shows that $D S$ is not necessary at all. So, once the validity of EXP is rejected, the proof of EXP from $D S$, mentioned at the beginning of this section, is not possible. Rather, it should be replaced by a derivation of $B$ that was the only alternative to obtain constructively $A \vee B$, given the impossibility of constructing $A$.

4.2. Ex quodlibet verum. Now, we turn to $E Q V$. Is the validity of the axiom 2.14 of Heyting (1956) indisputable in intuitionistic logic? As a matter of fact, we find no criticisms of $E Q V$ in the intuitionistic tradition. However, Johansson (1937) rejected the validity of explosion in intuitionistic logic because it seemed wrong to him to claim that when $\neg A$ has been proved, any $B$ at once becomes derivable from $A$, even when this had not been proved before (see Section 3.4). A similar but weaker argument could be used to cast doubt on $E Q V$, since it says that when $B$ has been proved, $B$ at once becomes derivable from any $A$, even when this had not been proved before.

The proof of $E Q V$ in a natural deduction system is as follows: 


$$
\frac{\frac{[A]^{1}}{B \rightarrow A} \rightarrow I}{A \rightarrow(B \rightarrow A)} \rightarrow I, 1
$$

The second application of $\rightarrow I$, that concludes $E Q V$, discharges the assumption $A$. But the first application of $\rightarrow I$ discharges an assumption that has not been declared. The introduction rule for implication allows the discharge of a vacuous assumption, and this move is tantamount to $E Q V$ in an axiomatic system. The rationale of this is that if a construction of $A$ is already given, any construction $B$, so to speak, can be 'placed on the top' of that construction of $A$ :

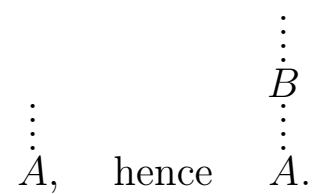

So, the construction of $A$ 'follows' from $B$ just because it was already there. We think, however, that this argument can be improved with the help of van Atten's interpretation $\beta$. First, note that reflexivity holds according to $\beta$. Given a condition $A$, the same condition $A$ is obtained, so, $A$ leads to $A$ :

$$
A \vdash A \text {. }
$$

We cannot see anything non-constructive in this reasoning. Now, given a condition $A$, any other condition $B$ added to $A$ still yields condition $A$, since anything that is obtained from $A$ can also be obtained from any $B$ that is put together with $A$. This is nothing but monotonicity, and there is no reason to suppose that constructive mathematical reasoning is non-monotonic. So, given reflexivity and monotonicity,

$$
A, B \vdash A
$$

holds, and two applications of the deduction theorem ${ }^{7}$ yield

$$
\vdash A \rightarrow(B \rightarrow A) \text {. }
$$

Again, we cannot see anything non-constructive in this line of reasoning.

4.3. The weak explosion. As we have seen, in the standard formalization of intuitionistic logic, the principle of explosion holds. In the formal systems proposed by Kolmogorov (1925) and Johansson (1937) (minimal logic), although EXP does not hold, a weaker principle, called here weak explosion, holds:

$$
(W-E X P) A, \neg A \vdash \neg B .
$$

It may seem that both EXP and $W$-EXP should be invalid in a Brouwerian intuitionistic logic because $W$-EXP is vulnerable to the same argument that justified the rejection of EXP in Section 2. Indeed, if there cannot be a constructive method that transforms the conditions on the construction of a contradiction into the conditions on the construction of an arbitrary proposition $B$, the same reasoning applies when $B$ is $\neg B$. However, this point is not so simple. The proof of $W$-EXP depends essentially on $E Q V$, which holds in intuitionistic logic and minimal logic. The proof of $W$-EXP is straightforward: from a pair of contradictory propositions $A$ and $\neg A$, from $E Q V$, we get $B \rightarrow A$ and $B \rightarrow \neg A$, and so, applying the introduction of negation and modus ponens, $\neg B$ is obtained. Similarly, if we take $\perp$ as primitive, from $E Q V$ we get

$$
\vdash \perp \rightarrow(B \rightarrow \perp)
$$

${ }^{7}$ It is not clear whether or not Kolmogorov (1925), Heyting (1930), Glivenko (1929) and Johansson (1937) had the deduction theorem - actually, it is likely that they didn't. However, the deduction theorem holds in all these formal systems. 
and so,

$$
\perp \vdash B \rightarrow \perp .
$$

What we have here is a sort of an aporetic situation: on one hand, a plausible argument that concludes the invalidity of EXP and can be extended to $W$-EXP; on the other hand, an even more plausible justification of $E Q V$.

4.4. On the normative character of $E X P$ and $W$-EXP. Although $W$-EXP does not make triviality tantamount to contradictoriness, a scenario in which all negated propositions can be inferred from any contradiction is not really any better than triviality. A logic in which EXP holds is called explosive, and a logic such that EXP does not hold but $W$-EXP holds is called partially explosive (Carnielli, Coniglio, and Marcos, 2007, p. 14).

Paraconsistent logics are formal systems in which the principle of explosion does not hold. A theory whose underlying logic is paraconsistent can accept contradictions without falling into triviality. It is a fact that several contexts of reasoning, from databases to scientific theories, are contradictory or yield contradictions in some critical situations. ${ }^{8}$ The motivation for paraconsistent logics is to have a formal system capable of dealing with such contexts in which contradictions are expected to occur. Clearly, paraconsistent logics cannot be partially explosive, and $W$-EXP cannot be valid. A paraconsistent logic in which $W$-EXP does not hold is called boldly paraconsistent (Carnielli, Coniglio, and Marcos, 2007, p. 14).

If EXP does not hold in a Brouwerian intuitionistic logic, the latter turns out to be a paraconsistent logic. However, this is not of much significance. Mathematicians, and Brouwer as well, work based on the assumption that mathematics is free of contradictions. The invalidity of EXP in a logic designed to express Brouwer's ideas is not because contradictions are possible, or eventually might appear. Indeed, Brouwer's remarks about non-contradiction (see Section 2.1 above) make it clear that a contradiction cannot be constructed. Besides, as we saw in Section 4.3 above, the argument for rejecting EXP in the intuitionistic framework is convincing, and a similar argument may be put forward against $W$-EXP. However, the rejection of $W$-EXP requires the rejection of $E Q V$, and the latter, in turn, as we have just seen, is a consequence of the deduction theorem, reflexivity and monotonicity.

The validity of $W$-EXP in intuitionistic logic may be explained from a normative point of view. Let us take a look at the role of EXP in classical logic. A central point of logic, no matter whether it is classical, intuitionistic or paraconsistent, is its normative character: the logical principles tell us how to reason correctly. In the case of intuitionistic logic, this normativeness comes not directly from logic, since the latter is a description of correct mental constructions. But once these principles are established, there is nothing wrong in taking them as normative. The classical notion of logical consequence is defined in terms of preservation of truth (of course, a transcendent notion of truth that is rejected by Brouwer). Hence, if one wants to reason correctly, in order to avoid obtaining false conclusion from true premises, one has to reason according to classical logic. The normative character of paraconsistent logic is clear: in a contradictory context of reasoning, EXP must not be applied, because although contradictions may occur, and do occur, triviality is unacceptable.

\footnotetext{
${ }^{8}$ See, for example, Belnap (1977) and Nickles (2002). Adopting a paraconsistent logic, however, does not imply the dialetheist view according to which there are true contradictions (e.g. Priest and Berto, 2013). The contradictions accepted by paraconsistent logics can be interpreted as conflicting evidence (Carnielli and Rodrigues, 2017) or inconsistent information (Belnap, 1977). In both cases, a property weaker than truth is attributed to contradictory propositions, in the sense that a proposition may enjoy that property without being true.
} 
The validity of EXP in classical logic is the result of a decision that took preservation of truth as both a necessary and sufficient condition for logical consequence. So, the justification of EXP in classical logic is as follows: preservation of truth is a sufficient condition for logical consequence, and there is no model $M$ such that both $A$ and $\neg A$ are true in $M$; therefore, for any $B$, there is no model $M$ such that $A$ and $\neg A$ are true in $M$ and $B$ is false in $M$. Although this justifies the validity of $E X P$, the role of $E X P$ in classical logic has not been explained yet. Of course, EXP is never used in classical reasoning, except for proving some other inferences - the disjunctive syllogism for example - that nevertheless already have an intuitive appeal independent from EXP. In our view, what explains the role of EXP in classical logic is its normative character. The principle of non-contradiction prohibits contradictions by saying that $A \wedge \neg A$ is always false. Explosion, in its turn, says something stronger: it explicitly states the consequences of accepting a contradiction. It is like two notices, one saying 'smoking not allowed here' and other saying 'smoking not allowed here - penalty: $£ 200$ '. Although from a strictly Kantian point of view, the consequences of the act should not interfere in the moral action, in fact - unfortunately maybe - the second notice is likely to be much more effective in avoiding smoking than the first. Something analogous occurs with respect to the principles of non-contradiction and explosion. The former just says that one cannot accept a contradiction because a contradiction is always false, while the latter says the consequences one is committed to if she/he accepts a contradiction. So, the role of EXP in classical logic is rather normative. It does not describe any real situation precisely because such a situation is not possible. Clearly, from the assumption that mathematics is free of contradictions, a pair of contradictory propositions can never be proved, either classically, or intuitionistically. So, it is reasonable to understand, and to explain, the validity of both EXP and $W$-EXP in a constructive logic designed to be a logic of constructive mathematical reasoning as a way of explicitly prohibiting contradictions.

\section{Final remarks}

In this paper, we discussed, from the point of view of Brouwer's conception of constructive mathematics, three principles that hold in the standard formulation of intuitionistic logic, namely, EXP (explosion, or ex falso quodlibet), $W$-EXP (weak explosion) and EQV (ex quodlibet verum). There are plausible reasons for rejecting EXP, and on this point we agree with van Atten (2009). But van Atten also defends the validity of disjunctive syllogism in a Brouwerian logic and suggests that the latter should be a relevance logic. In both cases, we do not agree with him. A relevance logic should reject EXP and $E Q V$. The same argument against EXP can be directed against $W$-EXP. But we saw in Section 4.3 that in order to reject $W-E X P$, since the latter follows from $E Q V$, at least one from reflexivity, monotonicity and the deduction theorem has to be considered invalid. In Section 4.2, we argued that these inferences should hold in a Brouwerian intuitionistic logic. Regarding disjunctive syllogism, van Atten's argument against it implies the loss of transitivity. In Section 4.1, we have shown that there is evidence in Brouwer's works that transitivity holds in constructive mathematics. We suggested also, in Section 4.4, that the validity of $W$-EXP in minimal logic may be understood with a normative role, even though this idea does not fit the descriptive character of logic defended by Brouwer.

Our conclusion here, however, is that although there are strong reasons for rejecting $E X P$, whether or not $E Q V$ and $W$-EXP should also be rejected in a Brouwerian intuitionistic logic is an issue not settled yet. More detailed investigations into Brouwer's work and into constructive results in mathematics are needed. 


\section{Bibliography}

N. Belnap. How a computer should think. In Contemporary Aspects of Philosophy (ed. G. Ryle). Oriel Press, 1977.

L. E. J. Brouwer. On the foundations of mathematics. In Collected Works vol. I. (ed. A. Heyting). North-Holland Publishing Company (1975), 1907.

L. E. J. Brouwer. The unreliability of the logical principles. In Collected Works vol. I. (ed. A. Heyting). North-Holland Publishing Company (1975), 1908.

L. E. J. Brouwer. Intuitionism and formalism. In Collected Works vol. I. (ed. A. Heyting). North-Holland Publishing Company (1975), 1912.

L. E. J. Brouwer. Consciousness, philosophy and mathematics. In Collected Works vol. I. (ed. A. Heyting). North-Holland Publishing Company (1975), 1948.

L. E. J. Brouwer. Points and spaces. In Collected Works vol. I. (ed. A. Heyting). NorthHolland Publishing (1975), 1954.

W. Carnielli and A. Rodrigues. An epistemic approach to paraconsistency: a logic of evidence and truth. Synthese, 2017. Preprint http://philsci-archive.pitt.edu/14115/1/ letj.pdf.

W. Carnielli, M. E. Coniglio, and J. Marcos. Logics of formal inconsistency. In Handbook of Philosophical Logic (eds. Dov Gabbay and Franz Guenthner), volume 14. Springer, 2007.

V. Glivenko. Sur la logique de M. Brouwer. Académie Royale de Belgique. Bulletin de la Classe des Sciences, 14:225-228, 1928.

V. Glivenko. On some points of the logic of Mr. Brouwer. In From Brouwer To Hilbert: The Debate on the Foundations of Mathematics in the 1920s (ed. P. Mancosu). Oxford University Press (1998), 1929.

S. Haack. Deviant Logic: Some Philosophical Issues. Cambridge University Press, 1974.

A. Heyting. The formal rules of intuitionistic logic. In From Brouwer To Hilbert: The Debate on the Foundations of Mathematics in the 1920s (ed. P. Mancosu). Oxford University Press (1998), 1930.

A. Heyting. Mathematische Grundlagenforschung, Intuitionismus, Beweistheorie. Springer-Verlag, 1934.

A. Heyting. Les Fondements des Mathématiques, Intuitionnisme, Théorie de la Démonstration. Paris: Gautier-Villars, 1955.

A. Heyting. Intuitionism: an Introduction. North-Holland Publishing Company, 1956.

D. Hilbert. The logical foundations of mathematics. In From Kant to Hilbert: A Source Book in the Foundations of Mathematics (ed. William Ewald), volume 2. Oxford University Press (1996), 1923.

I. Johansson. Der Minimalkalkül, ein reduzierter Intuitionisticher Formalismus. Compositio Mathematica, pages 119-136, 1937.

A. Kolmogorov. On the principle of excluded middle. In From Frege to Gödel (ed. Jan van Heijenoort). toExcel Press (1979), 1925.

A. Kolmogorov. On the interpretation of intuitionistic logic. In From Brouwer To Hilbert: The Debate on the Foundations of Mathematics in the 1920s (ed. P. Mancosu). Oxford University Press (1998), 1932. 
E. Mares. Relevance logic. The Stanford Encyclopedia of Philosophy (Ed. E. Zalta), 2018. T. Nickles. From Copernicus to Ptolemy: inconsistency and method. In Inconsistency in Science (Ed. J. Meheus). Dordrecht: Springer, 2002.

G. Priest and F. Berto. Dialetheism. Stanford Encyclopedia of Philosophy, 2013.

N. Tennant. Natural Deduction and Sequent Calculus for Intuitionistic Relevant Logic. The Journal of Symbolic Logic, 52(3):665-680, 1987.

A. S. Troelstra and D. van Dalen. Constructivism in Mathematics: an Introduction. Elsevier, 1988.

M. van Atten. On the hypothetical judgement in the history of intuitionistic logic. In Logic, Methodology, and Philosophy of Science: Proceedings of the Thirteenth International Congress (eds. C. Glymour, W. Wei, and D. Westerstahl). College Publications, 2009.

M. van Atten. The development of intuitionistic logic. The Stanford Encyclopedia of Philosophy, 2017.

D. van Dalen. Kolmogorov and Brouwer on constructive implication and the Ex Falso rule. Russian Mathematical Surveys, 59:247-257, 2004.

D. van Dalen. Another look at Brouwer's dissertation. In One Hundred Years of Intuitionism (ed. M. van Atten et al.). Birkhäuser Verlag, 2008.

D. van Dalen, editor. The Selected Correspondence of L. E. J. Brouwer. Springer, 2011. 